\title{
SUSTAINABLE PROCUREMENT IN HEALTHCARE
}

\author{
Susan Wilburn, Ishika Jharia and Poornima Prabhakaran
}

\section{Introduction}

Sustainable and climate-friendly healthcare encompasses activities and services across the entire lifecycle of healthcare delivery. This includes the upstream health sector supply chain which represents the largest proportion of a health system's greenhouse gas emissions. Greenhouse gas emissions are divided into scopes 1,2 and 3, as outlined in the chapter titled The Role of Health Systems in the Global and Indian Context. Supply chain emissions mostly fall under scope 3 which include: purchases, products and services (including transportation and the distribution of products), patient transportation and employee commuting and waste management (WRI, 2020). In India, scope 3 emissions represent $81 \%$ of the total health sector emissions (Karliner et al., 2019). India serves as a critical component of the global supply chain as the largest supplier of goods provided to the UN. It is, therefore, important for the Indian health sector to measure, monitor and act to reduce greenhouse gas emissions from throughout the health supply chain.

Sustainable health systems are those that deliver healthcare based on not just the economic sustainability of their own operations but also on the impacts of those services on the environment. Sustainable Procurement stands on the three principles of environmental, social and economic sustainability as described in Goal 12 of the United Nations' Sustainable Development Goals: sustainable production and consumption (UN, 2015). In sustainable procurement, organizations meet their needs for goods and services while generating benefits for the organization, society and the economy while minimising damage to health and the environment. Sustainable procurement looks at how to achieve value on a whole-life basis. This chapter will:

1. Review the greenhouse gas emissions of the health supply chain and provide an overview of the guiding principles for fostering environmental sustainability by promoting sustainable procurement in the healthcare sector. 
2. Describe how the sustainable procurement principles and methods identified will guide contributions by health sector actors to achieving transition to sustainable alternatives for health sector procurement.

3. Provide case studies from India and England as examples of sustainable procurement in the health sector.

\section{The climate footprint of the health sector in India}

The health sector emissions in India are equivalent to the emissions of 10 coal-fired power plants or 5,16,286 tanker trucks worth of gasoline or 8,280,255 passenger vehicles for a year (HCWH and Arup, 2019, p. 26). Taking emissions from various sectors into account, India's healthcare sector's contribution to total carbon emissions is $1.5 \%$ as compared to the global average of $4.4 \%$.

If the global health sector were a country, it would be the fifth-largest greenhouse gas emitter on the planet. More than half of the sector's carbon footprint comes from its supply chain. Fossil fuel consumption is at the heart of healthcare's emissions through its energy consumption across the life cycle of its operations. The lion's share of emissions (71\%) is primarily derived from the healthcare supply chain through the production, transport, packaging, transport and disposal of medical devices, pharmaceuticals and other products. Emissions emanating directly from healthcare facilities and healthcare owned vehicles make up $17 \%$ of the sector's worldwide footprint (HCWH and Arup, 2019, p. 5).

\section{Principles guiding sustainable health systems}

As outlined above, healthcare including hospitals, health systems and the health products' supply chain can paradoxically contribute to emissions from the entire lifecycle of their operations. The sector therefore has a responsibility to adopt sustainable, low-carbon solutions to mitigate and reduce their own climate footprint while incorporating the principles of environmental, economic and social responsibility to forge parallel and related paths towards net zero carbon emissions. The following section outlines some key principles guiding this action:

\section{A. Environmental sustainability}

Key steps towards environmental sustainability can be based on the Greenhouse Gas Protocol and should include:

SCOPE 1 - Taking immediate action to reduce healthcare facility emissions (Box 18.1).

SCOPE 2 - Investing in and advocating for the decarbonisation of local and national energy systems and the implementation of clean, renewable energy. 
SCOPE 3 - Setting and implementing criteria for low-carbon or zeroemissions procurement so as to begin to decarbonise the supply chain.

\section{Box 18.1 Case study}

Potential sustainable options: Health Care Without Harm, a global organization working to help health systems around the world move to sustainable healthcare practices has identified the following five high impact, low carbon interventions for health sector action:

- Transition to renewable forms of energy

- Energy-efficient low hydro-fluorocarbon (HFC) air conditioners and cooling technologies

- Intravenous anaesthetic agents, closed anaesthetic systems, elimination of desflurane and minimisation of nitrous oxide

- Energy-efficient lighting (LEDs) and optimisation of the use of natural light

- Low/zero-emission transportation and fleet vehicles

The adoption of a framework to implement these interventions as short-term goals across health systems will provide a starting point for decarbonising healthcare facilities.

\section{B. Economic principles: the significance of whole life/lifecycle costing in sustainable procurement}

Whole-life costing takes account of the total cost of a product or service over its lifetime, from determining the need for it, through to its eventual disposal and replacement. For example, for equipment, it would include the cost of maintaining and operating the product as well as outright purchase, hire or lease price; the cost of consumables, utilities, training; and the cost of disposal or potential sale value at the end of its life (Figure 18.1).

\section{Social criteria in sustainable procurement}

In addition to principles of environmental sustainability, social criteria must also be addressed for a holistic approach to procurement. Social sustainability consists of stakeholder engagement, community cooperation, a people-centred approach to organizational procurement impacts and inclusive social development in terms of health and safety standards, local production and ethically sourced goods and services. 


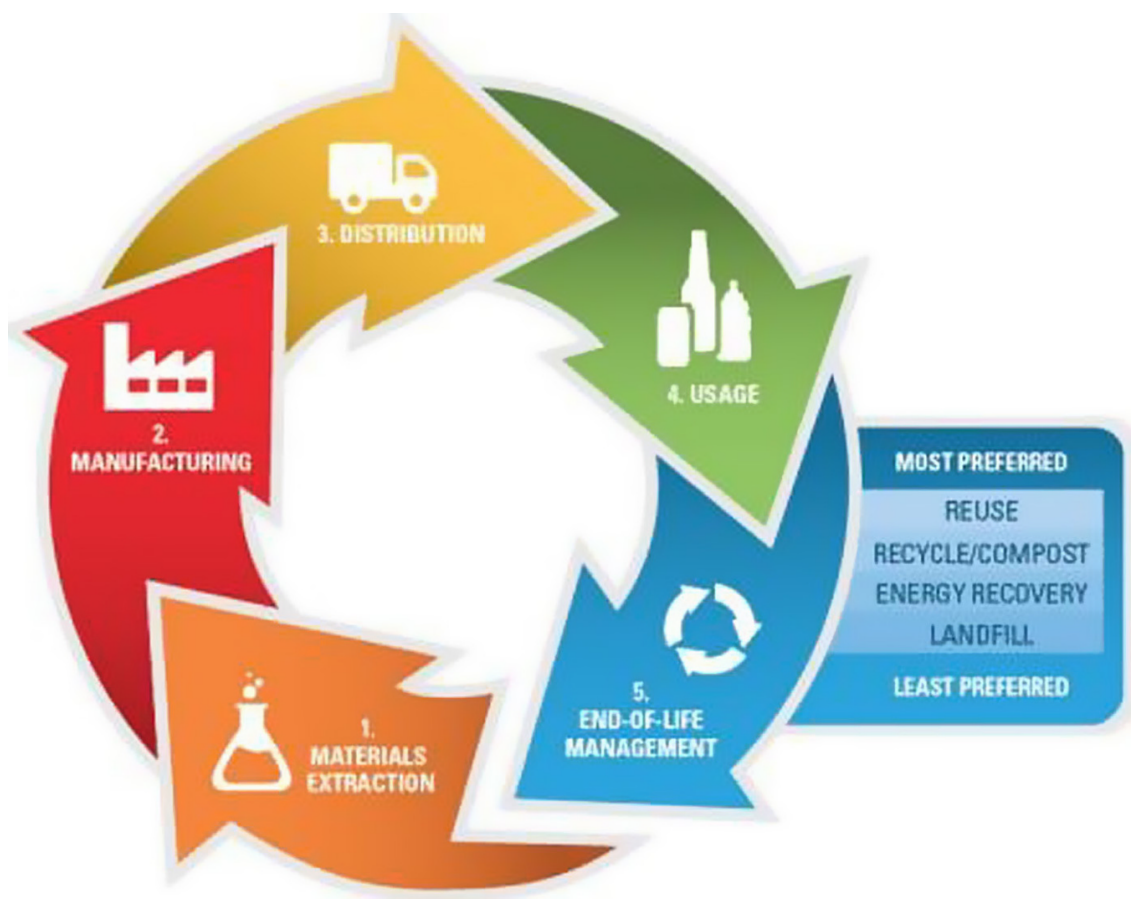

Figure 18.1 Lifecycle of materials and products from material extraction, manufacturing, distribution and use till end-of-life.

Source: USEPA, 2017

\section{Examples of sustainable procurement practices in India}

The application of the above principles of reducing sectoral emissions, lifecycle costing and social principles enables the right sustainable purchasing practices. The incorporation of various criteria in procurement of general goods has been practiced in India in the past. For example, principles of star rating appliances are established by the Bureau of Energy Efficiency (BEE). The procurement of LED bulbs, solar panels and certain star rated appliances is mandated in some government agencies. The Central Public Works Department (CPWD) in its tender conditions state that buildings constructed shall follow the Green Rating for Integrated Habitat Assessment (GRIHA) principles. Many state governments have taken initiatives to install off-grid solar photovoltaic systems in their respective state rural health centres, funded by either a mix of state (usually health department) and central government support, or entirely through the state budget; and in certain cases, through partnership between government ministries and international organizations. For example, the Chhattisgarh State Health Department collaborated with the Chhattisgarh State Renewable Energy 
Development Agency (CREDA) on a programme to provide solar power at all the primary health centres (Ramji et al., 2017).

These principles do ensure environmental sustainability; however, with the above initiatives already in place, there is also the need to bring the focus on procuring sustainable alternatives for goods and consumables in healthcare. Two examples are the procurement of digital thermometers and sphygmomanometers in place of mercury thermometers and sphygmomanometers, and nitrile gloves in place of Polyvinyl Chloride (PVC) gloves. These changes were made in order to replace polluting and potentially toxic materials with sustainable alternatives.

Such transitions to sustainable alternatives can also be practiced in the case of sterilants and disinfectants. The most common high-level disinfectants are glutaraldehyde and formaldehyde, and cold sterilant ethylene oxide. Formaldehyde and glutaraldehyde cause breathing discomfort to the users, and ethylene oxide and formaldehyde are known human carcinogens (IARC data, 2020). Safer alternatives that exist include: peracetic acid, hydrogen peroxide, steam sterilisation, UV radiation, etc. Similarly, a conscious move to better-managed procurement of pharmaceuticals, medical devices, business products and services, food/catering, and other health facility inputs are key to the efficient and sustainable functioning of healthcare facilities.

In order to facilitate a global practice within the Indian healthcare system of sustainable procurement, guidelines to sustainable procurement are being incorporated within national and sub-national programmes including the Indian Public Health Standards (IPHS) and the National Program for Climate Change and Human Health. The current advocacy for developing all levels of Indian healthcare into green and resilient facilities incorporates an entire vertical of sustainable procurement.

The following section provides two case studies where sustainable practices were adopted in healthcare (Boxes 18.2 and 18.3).

\section{Recommendations}

Sustainable procurement of goods and services for healthcare must address the entire lifecycle of healthcare delivery, spanning the manufacture and supply of goods such as pharmaceutical products and medical devices, the actual delivery of healthcare with efficient and sustainable procurement of services for resources like water and energy, and appropriate disposal of used products at the end of life, including waste, packaging materials, food waste, etc. The incorporation of appropriate technical specifications in tender documents for the sustainable procurement of goods is a strategic way to create demand for the production of such goods. Sustainable Procurement in Health Care Guide, a useful resource recently released by Health Care Without Harm, provides a step-by-step guide to sustainable procurement in healthcare (HCWH and GGHH, 2020). 


\section{Box 18.2 Case study}

\section{NHS England procurement for carbon reduction}

The Sustainable Development Unit (SDU) of the National Health Service (NHS) in England has conducted the most extensive analysis of the carbon footprint of their supply chain, identified the hot spots in their supply chain and analysed the interventions that are the most cost-effective in order to reduce carbon and save money.

Figure 18.2 shows the detailed break-down of the products and services that represent the top carbon emissions of the NHS (NHS, 2012). In acute (hospital) care, the top 3 carbon emissions are biomedical instruments/equipment and building energy use (gas and electricity). The procurement of pharmaceutical agents represents the highest emissions from primary care settings in England. The NHS SDU worked with their largest suppliers of pharmaceuticals to develop and build consensus around measuring carbon emissions within the drug supply chain. Within pharmaceuticals, waste anaesthetic gas represented $5 \%$ of the NHS acute care emissions, and metered-dose inhalers for the treatment of asthma represented 3.5\% of the total carbon footprint of the NHS (Public Health England and NHS SDU, 2018). The NHS Procurement for Carbon Reduction (P4CR) programme includes a hierarchy of four interventions to reduce the emission of the health system:

1) Reducing the demand for products and services

2) Reducing the in-use emissions such as reduced energy use and improved energy efficiency (for example, the procurement of LED energy efficient lightbulbs)

3) Innovation and substitution

4) Supply chain management

The NHS has also identified the most cost-effective and cost-saving GHG reduction interventions in a Marginal Abatement Curve (MAC). Changes in the heating and ventilation system to become more efficient, and the substitution of dry powdered inhaler asthma medicine to replace the potent greenhouse gas emissions from metered-dose inhalers, are among the interventions that save money and reduce carbon emissions. 


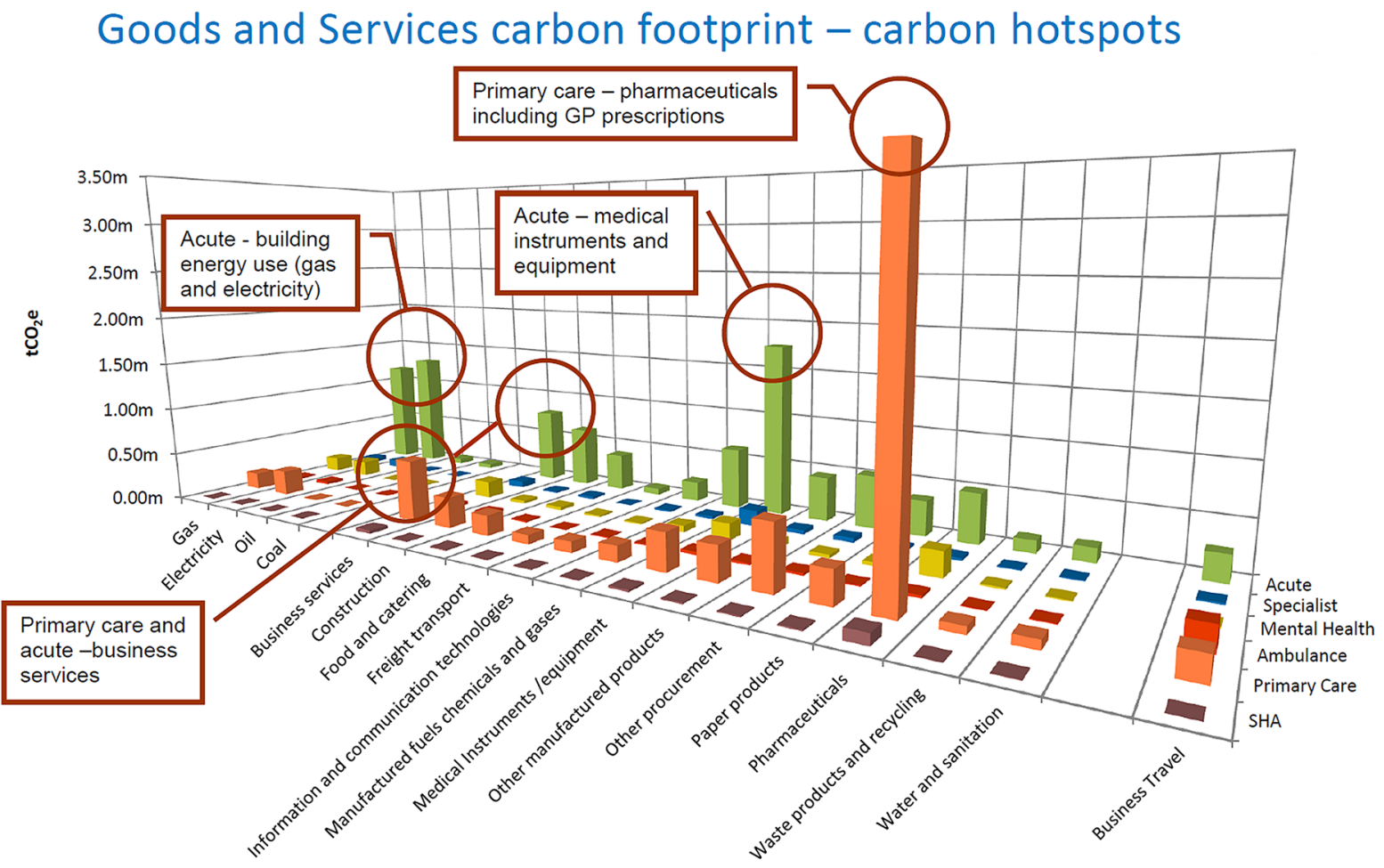

Figure 18.2 NHS and social care England goods and services' carbon footprint - carbon hotspots.

Source: National Health Service, England: Sustainable Development Unit 


\section{Box 18.3 Case study}

\section{A solar energy initiative at Dr. R. N. Cooper Municipal General Hospital, Mumbai}

HBT Medical College and Dr. R. N. Cooper Hospital is a 636-bedded secondary care municipal hospital with all general specialties, located in the western suburbs of Mumbai. Large in size and capacity, the hospital addresses diverse needs that require round-the-clock use. This means inevitable energy use which often results in high and overlooked consumption. Motivated to reduce operating costs and support sustainability goals, the management team at the municipal general hospital decided to install passive solar thermal water heating systems with a capacity of $3000 \mathrm{~L}$ per day (each) on six buildings within the hospital campus.

The total installation cost of the solar thermal water heating systems was $4,230,000$ INR $(\$ 58,570)$. The time for the return on investment of this system was calculated at 19 months. 100L of water per day, heated from this system, saves up to 1500 electricity units annually as per the efficiency capacity installed. Therefore, an 18,000 L per day system saves 27,0000 electricity units per year. The cost of one unit of electricity is $10 \mathrm{INR}$, resulting in a savings of 2,700,000 INR $(\$ 37,500)$ net savings per year. In terms of carbon savings and environmental benefits, an 18,000 L per day system saves 27,000 electricity units per year, which in turn reduces 270 tonnes of $\mathrm{CO} 2$ emission into the atmosphere; whereas to generate 1500 units of electricity per year from a coal-based power plant, 1.5 tonne of $\mathrm{CO}_{2}$ is released into the atmosphere.

Dr. RN Cooper hospital aims for more energy efficiency to follow; however, it is recommended to focus first on efficiency before procuring anything new. There are many easy low and no-cost options to help save on energy expenses and improve energy performance. Simple measures such as switching off all non-essential lights, turning off unused electrical and office equipment, putting x-rays on standby, and using natural ventilation methods for cooling, can have considerable financial, environmental and strategic benefits.

Source: Global Green and Healthy Hospitals, 2018

\section{Key takeaways}

- Health systems that aspire to carbon neutrality must analyse their supply chain, identify carbon hot spots and engage with suppliers to establish targets for the reduction of greenhouse gas emissions throughout 
the life cycle of the products from manufacturing to distribution to end of use/reuse.

- Procurement policies that integrate sustainability criteria for a lowcarbon health supply chain are needed to achieve the goal of climatesmart, resilient health systems.

\section{References}

Global Green and Healthy Hospitals. (2018). A solar energy initiative to reduce cost. https:/www.ceh.org.in/wp-content/uploads/2019/01/GGHH-Case-StudyEnergy_RN-Cooper_AT_SM.pdf.

HCWH and Arup. (2019). Healthcare's climate footprint: How the health sector contributes to the global climate crisis and opportunities for action. HCWH and Arup. https://noharm-global.org/sites/default/files/documents-files/5961/ HealthCaresClimateFootprint_092319.pdf.

HCWH and GGHH. (2020). Sustainable procurement in health care guide. Practice Greenhealth.

International Agency for Research on Cancer (IARC). (2020). Monographs on the identification of carcinogenic hazards to bumans. https://monographs.iarc.fr/ agents-classified-by-the-iarc/ 26 July 2020.

Karliner, J., Slotterback, S., Boyd, R., Ashby, B., \& Steele, K. (2019). Health care's climate footprint: How the health sector contributes to the global climate crisis and opportunities for action. Health Care Without Harm and ARUP.

National Health Service (NHS - England) Sustainable Development Unit (SDU). (2012). Goods and services carbon hotspots. https://www.sduhealth.org.uk/ policy-strategy/reporting/natural-resource-footprint-2018/carbonhotspots.aspx.

Public Health England and NHS SDU. (2018). Natural resource footprint: Reducing the use of natural resources in health and social care. https://www.sduhealth.org. uk/policy-strategy/reporting/naturalresource-footprint-2018.aspx.

Ramji, A., Patnaik, S., Mani, S., \& Dholakia, H. H. (2017). Powering primary healthcare through solar in India: Lessons from Chhattisgarh. Council on Energy, Environment and Water. https://www.ceew.in/publications/powering-primaryhealthcare-through-solar-india

United Nations. (2015). UN sustainable development goals. www.un.org/sustainabledevelopment/sustainable-development-goals/.

USEPA. (2017). Sustainable materials management basics. https://www.epa.gov/ $\mathrm{smm} /$ sustainable-materials-management-basics\#lifecycle.

World Resources Institute. (2020). Greenhouse gas protocol. www.ghgprotocol.org. 